\title{
Correlation between visual acuity and human leukocyte antigen DRB1*04 in patients with Vogt-Koyanagi-Harada disease
}

\author{
Norihiko Misawa, Mizuki Tagami", Takeya Kohno and Shigeru Honda
}

\begin{abstract}
Background: The common presence of human leukocyte antigen (HLA)-DRB1*04 in Vogt-Koyanagi-Harada (VKH) disease is well known. The aim of this study was to investigate the relationship between visual prognosis and HLA-DRB1*04 alleles during systemic corticosteroid therapy in patients with VKH disease.

Methods: This retrospective case series included 57 eyes from 29 consecutive patients with treatment-naive VKH disease who received systemic corticosteroid therapy. Visual acuity, sex, refractive error, central retinal thickness (CRT), central choroidal thickness $(C C T)$, and duration from onset to treatment were measured at initial and final visits. Mean values of parameters were compared with each visit. Genotyping was performed by polymerase chain reaction amplification with sequence-specific primer.

Results: Linear regression showed significant differences in logMAR best-corrected visual acuity between the three groups of homozygotes, heterozygotes, and normal subjects at baseline $(p<0.01)$, at 3 months after treatment $(p<0.01)$. There was no significant differences at 6 months after treatment $(p=0.257)$. No significant differences were detected between the three groups in age, sex, refractive error, CRT, CCT, or duration from onset to treatment.
\end{abstract}

Conclusion: Alleles of HLA-DRB1*04 might affect visual prognosis and be related to early response after initiation of treatment in VKH disease.

Keywords: Vogt-Koyanagi-Harada disease, HLA-DRB1*04, Japanese patients

\section{Background}

Vogt-Koyanagi-Harada (VKH) disease is a systemic disorder that is considered to represent an autoimmune disease against melanocytes [1]. In the eye, the disease presents as acute bilateral granulomatous panuveitis, which responds to systemic corticosteroid therapy and generally shows good visual prognosis with relatively short follow-up [2]. A role of genetic factors such as HLA alleles in the development of VKH was first considered in 1976, and was supported by the simultaneous development of $\mathrm{VKH}$ in monozygotic twins [3]. The human leukocyte antigen (HLA) system is the locus of

\footnotetext{
* Correspondence: tagami.mizuki@med.osaka-cu.ac.jp Department of Ophthalmology and Visual Science, Graduate School of Medicine, Osaka City University, 1-5-7 Asahimachi, Abeno-ku, Osaka-shi 545-8586, Japan
}

genes that encode for major histocompatibility complex (MHC), representing a set of cell surface molecules mediating the interaction of leukocytes [4]. HLA, and HLADRB1*04 in particular, therefore plays a key role in immune systemic function, as well as in the pathogenesis of autoimmune diseases, including VKH [5]. A Korean study found that the HLA DRB1*0405 allele conferred an increased relative risk of developing $\mathrm{VKH}$ compared with the general population, and that the HLA DRB1*0405-DQA1*0302-DQB1*0401 haplotype was associated with poorer visual prognosis [6]. On the other hand, despite systemic corticosteroid approach, VKH patients often prove refractory to systemic corticosteroid therapy with long follow-up $[7,8]$. For predicting prognosis and refractoriness to treatment, the focus in recent years has transitioned from the identification of HLA 
genes associated with increased risk of VKH to the identification of alternate genes $[9,10]$. However, in Japan, HLA has traditionally been examined for uveitis, and accumulated data are available for our facilities. For that reason, we report an investigation of correlations between HLA04 allele type and visual outcomes before and after steroid treatment in the real world.

\section{Methods}

This retrospective case series included 57 eyes from 29 consecutive patients with treatment-naïve VKH disease who visited the ophthalmology department at Osaka City University Hospital between December 2009 and January 2019 and were followed up for more than 6 months after the start of systemic corticosteroid therapy. VKH disease was diagnosed according to the criteria of Sugiura [11] and the VKH Disease Committee [12]. None of the patients had any medical or ocular history at the initial visit. Changes in central retinal thickness (CRT) and central choroidal thickness (CCT) were assessed by vertically and horizontally oriented enhanced depth imaging optical coherence tomography (EDI-OCT) of the macula (Spectralis HRA + OCT Heidelberg Engineering, Heidelberg, Germany) for up to 3 months after treatment. Measurements of CRT and CCT in each eye were then reconfirmed by three experts (NM, MT, and $\mathrm{SH}$ ) by checking the OCT images. Patients received corticosteroid regimens of pulse therapy according to the timing of their first visit. Recurrences were defined as the recurrence of anterior chamber cells and/or posterior segment lesions detected by ophthalmic examinations.

In HLA typing by polymerase chain reaction amplification with sequence-specific primers (PCR-SSP), typing specificity is part of the amplification step [9].

The Kruskal-Wallis test and Fisher's exact test were used to analyze and compare baseline patient characteristics. The relationship between HLA04 allele and visual outcomes was determined using univariate linear regression analysis. Analysis of covariance was used to analyze final visual acuity to clarify the influence of baseline visual acuity. In addition, in the case of regression analysis, an explanatory variable that roughly divided the number of cases by 15 was considered appropriate [13].

Statistical analyses were performed using SPSS Statistics version 22 software (IBM Japan, Tokyo, Japan). Values of $P<0.05$ were considered statistically significant.

\section{Results}

\section{Treatment and patient characteristics}

Patients received regimens of corticosteroid pulse therapy according to the timing of their first visit. In the 27 patients administered intravenous methylprednisolone, the dose was $1000 \mathrm{mg} /$ day for 3 consecutive days followed by tapering of oral prednisolone (i.e., pulse therapy), as described previously [14]. Oral prednisolone was temporarily increased or restarted in the event of anterior or posterior recurrence of $\mathrm{VKH}$ disease. The remaining two patients were administered intravenous prednisolone and tapered from $100 \mathrm{mg} /$ day (i.e., high-dose therapy), as described previously [7]. Oral cyclosporine was not administered in the present study. We calculated duration from onset to steroid administration and duration from first visit to steroid administration in all patients. Mean duration from onset to steroid administration was $17.4 \pm$ 10.7 days and mean duration from first visit to steroid administration was $4.1 \pm 3.2$ days. Baseline patient characteristics are summarized in Table 1 . The mean follow-up was $22 \pm 20$ months (range 6-105 months). Recurrences of inflammation were observed in 5 patients (17\%) over follow-up, and they consisted of visible posterior segment inflammation (subretinal fluid or choroidal white lesions) in all patients.

\section{Visual outcomes}

In visual acuity, mean $\log M A R$ best-corrected visual acuity (BCVA) values at baseline, at 3 months after treatment and, at 6 months after treatment were $0.34 \pm 0.58,0.01 \pm 0.25$, and $0.01 \pm 0.38$ in HLA-DRB1*04 $-/-,-0.16 \pm 0.33,-0.11 \pm$ 0.06 , and $-0.06 \pm 0.14$ in HLA-DRB1*04 $+/-$, and $0.008 \pm$ $0.14,-0.13 \pm 0.05$, and $-0.09 \pm 0.24$ in HLA-DRB1*04 $+/+$, indicating significant visual improvements from baseline after treatment in HLA-DRB1*04 + $/$ - and HLA-DRB1*04 $+/$ +group (3 M: $p<0.01, p<0.01, p=0.056$, respectively) (6 $\mathrm{M}: p=0.24, p<0.01, p=0.07$,respectively). In covariate analysis considering the influence of baseline visual acuity, the HLA-DRB1*04 - / - and HLA-DRB1*04 +/- groups showed significant differences in the final visit $(p<0.01)$. Mean CCT values pretreatment and at final visit after treatment were $489.4 \pm 258.4$ and $285.2 \pm 159.4 \mu \mathrm{m}$ in HLA-DRB1*04 $-/-$, $557.5 \pm 197.5$ and $356.1 \pm 135.5 \mu \mathrm{m}$ in HLA-DRB1 ${ }^{*} 04+/$-, and $517.5 \pm 190.5$ and $375.8 \pm 211.2 \mu \mathrm{m}$ in HLA-DRB1*04 $+/+$, indicating changes compared with baseline $(p=0.110$, $p<0.01, p=0.173$, respectively). In the HLA-DRB1*04 $+/-$ group alone, mean post-treatment CCT values were significantly decreased compared with baseline CCT.

\section{Correlation with HLA-DRB $1 * 04$ allele}

HLA-DRB1*04 typing was performed for 5 patients (10 eyes) in normal subjects (HLA-DRB1*04-/-), 15 patients $(29$ eyes) in heterozygotes (HLA-DRB1*04 $+/-$ ), and 6 patients (12 eyes) in homozygotes (HLA-DRB1*04 $+/+)$. Linear regression analysis showed significant differences among the three groups of homozygotes, heterozygotes, and normal subjects in logMAR BCVA at baseline in Fig. $1(p<0.01)$.

Comparisons of clinical parameters at post-treatment in the three groups are summarized in Table 2. Linear 
Table 1 Pre-treatment parameters baseline patient characteristics

\begin{tabular}{lllll}
\hline HLA-DRB1*04 allele type & HLA-DRB1*04 $-/-$ & HLA-DRB1*04 +/- & HLA-DRB1*04 +/+ & $p$ \\
\hline Age (years) & $47.83 \pm 23.78$ & $50.73 \pm 17.53$ & $49.52 \pm 14.24$ & $3: 5$ \\
Sex (male:female) & $3: 3$ & $9: 5$ & $-2.23 \pm 2.74$ & $0.47^{* *}$ \\
Refractive error (diopters) & $-0.93 \pm 17.15$ & $-3.31 \pm 2.99$ & $517.5 \pm 190.5$ & $0.81^{*}$ \\
CRT $(\mu \mathrm{m})$ & $489.4 \pm 258.4$ & $557.5 \pm 197.5$ & $518 \pm 191$ & $0.86^{*}$ \\
CCT $(\mu \mathrm{m})$ & $210 \pm 297$ & $310 \pm 317$ & $19.25 \pm 9.48$ & $0.86^{*}$ \\
Duration from onset to treatment (days) & $18.7 \pm 12.68$ & $16.07 \pm 11.20$ & $0.58^{*}$ \\
\hline
\end{tabular}

CRT central retinal thickness, $C C T$ central choroidal thickness

* Kruskal-Wallis test

** Fisher's exact test

regression analysis found significant differences among the three groups of homozygotes, heterozygotes, and normal subjects in logMAR BCVA at 3 months after treatment in Fig. $2(p<0.01)$. There was no significant differences at 6 months after treatment $(p=0.25)$. This result was consistent with the finding of no difference among three groups with regard to choice of treatment regimen according to the period during which the patient visited the clinic (i.e., large dose, earlier; pulse, later). Second, the number of recurrences during followup did not differ between the three groups.

In terms of visual prognosis of the 57 eyes examined, we documented mild to moderate cataracts in 4 eyes and drug-controllable glaucoma in 3 eyes after treatment, none of which required surgery during follow-up. No differences in the frequency of these ocular complications were seen among patients.

\section{Discussion}

The present study revealed a correlation between HLA04 allele and visual outcomes before and after initiating systemic corticosteroid therapy in treatment-naïve patients with VKH disease. In this study, linear regression showed significant differences in logMAR BCVA at baseline and at 3 months after treatment between the three groups of homozygotes, heterozygotes, and normal subjects. There were no significant differences at 6 months after treatment. We can guess that Alleles of HLA-DRB1*04 might affect visual prognosis and be related to early response after initiation of treatment in VKH disease. From these results. Also, although the statistical study did not yield significant results, we speculate that CRT and CCT might show significant differences with greater numbers of cases of homozygotes, heterozygotes, and normal subjects. These thicknesses may be associated with the final prognosis of the disease [14]. The duration from first visit to steroid administration was very short. This was taken as a likely reason for the low recurrence rate for VKH compared with other reports without the use of immunosuppression therapy [7]. Cyclosporine was approved in 2012, and Adalimumab was also approved by the government for several years. But not all recommended approaches are government approved in Japan. For these reasons, steroid-centered

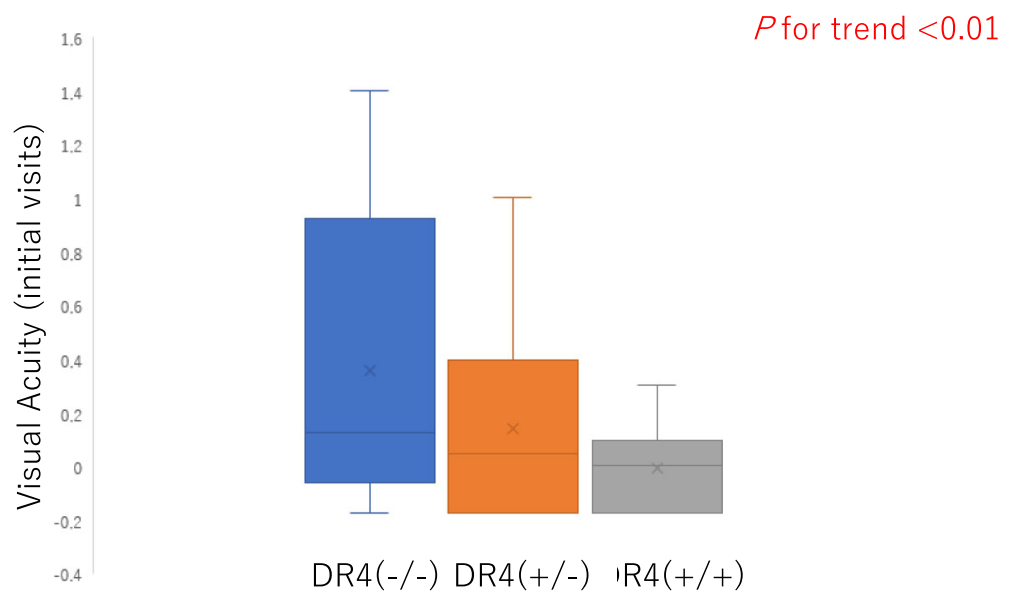

Fig. 1 Linear regression for best-corrected visual acuity (BCVA) at baseline. LogMAR BCVA for Vogt-Koyanagi-Harada (VKH) disease at baseline on linear regression among the three groups of homozygotes (HLA-DRB1*04 $+/+$ ), heterozygotes (HLA-DRB1*04 $+/-$ ), and normal subjects (HLA-DRB1*04 -/-) 
Table 2 Clinical parameters compared post-treatment among the three groups

\begin{tabular}{|c|c|c|c|c|c|c|c|c|}
\hline & Patient & $\begin{array}{l}\text { Duration from onset } \\
\text { to treatment(days) }\end{array}$ & $\begin{array}{l}\text { Duration from first visit } \\
\text { to treatment(days) }\end{array}$ & Recurrence & $\begin{array}{l}\text { Post-treatment } \\
\text { ocular complications }\end{array}$ & $\begin{array}{l}\text { Initital } \\
\text { treatment }\end{array}$ & $\begin{array}{l}\text { Final logMAR } \\
\text { BCVA R }\end{array}$ & $\begin{array}{l}\text { Final logMAR } \\
\text { BCVA L }\end{array}$ \\
\hline \multirow{8}{*}{$\begin{array}{l}\text { HLA- } \\
\text { DRB1*04 } \\
+/+\end{array}$} & 9 & 37 & 11 & none & none & paluse & -0.176091259 & $-\overline{0.176091259}$ \\
\hline & 11 & 23 & 2 & none & none & paluse & -0.176091259 & 0 \\
\hline & 13 & 14 & 1 & $\begin{array}{l}\text { PSL } \\
\text { increased }\end{array}$ & none & $\begin{array}{l}\text { STTA(40 } \\
\text { mg),paluse }\end{array}$ & -0.079181246 & -0.176091259 \\
\hline & 16 & 11 & 9 & none & none & paluse & -0.176091259 & $\overline{-} .176091259$ \\
\hline & 20 & 12 & 1 & none & none & paluse & -0.176091259 & -0.176091259 \\
\hline & 23 & 25 & 2 & none & Left cataract & paluse & -0.176091259 & -0.176091259 \\
\hline & 25 & 9 & 4 & none & none & paluse & -0.079181246 & $\overline{-} .079181246$ \\
\hline & 27 & 23 & 8 & none & none & paluse & -0.079181246 & $\overline{-} .079181246$ \\
\hline \multirow{15}{*}{$\begin{array}{l}\text { HLA- } \\
\text { DRB1*04 } \\
+/-\end{array}$} & 2 & 7 & 2 & none & none & paluse & 0 & -0.079181246 \\
\hline & 4 & 14 & 6 & none & none & $\begin{array}{l}\mathrm{STTA}(40 \\
\mathrm{mg}) \text {,paluse }\end{array}$ & -0.176091259 & -0.176091259 \\
\hline & 5 & 40 & 2 & $\begin{array}{l}\text { PSL } \\
\text { increased }\end{array}$ & Left cataract & paluse & -0.079181246 & -0.079181246 \\
\hline & 8 & 19 & 5 & none & none & paluse & -0.176091259 & -0.176091259 \\
\hline & 10 & 12 & 7 & none & none & paluse & & 0.301029996 \\
\hline & 12 & 21 & 0 & $\begin{array}{l}\text { PSL } \\
\text { increased }\end{array}$ & none & paluse & 0 & -0.079181246 \\
\hline & 14 & 39 & 6 & $\begin{array}{l}\text { PSL } \\
\text { increased }\end{array}$ & none & paluse & -0.079181246 & -0.079181246 \\
\hline & 17 & 6 & 1 & $\begin{array}{l}\text { PSL } \\
\text { resumed }\end{array}$ & none & $\begin{array}{l}\text { PSL40mg, } \\
\text { paluse }\end{array}$ & 0 & -0.079181246 \\
\hline & 18 & 11 & 4 & none & none & paluse & -0.176091259 & -0.079181246 \\
\hline & 19 & 6 & 1 & none & none & paluse & -0.176091259 & 0 \\
\hline & 21 & 8 & 1 & none & none & paluse & -0.176091259 & -0.176091259 \\
\hline & 22 & 23 & 1 & none & none & paluse & -0.176091259 & -0.176091259 \\
\hline & 26 & 3 & 5 & none & none & paluse & -0.176091259 & -0.176091259 \\
\hline & 28 & 14 & 12 & none & none & paluse & -0.176091259 & -0.176091259 \\
\hline & 29 & 18 & 6 & none & none & paluse & -0.176091259 & -0.176091259 \\
\hline \multirow{6}{*}{$\begin{array}{l}\text { HLA- } \\
\text { DRB1*04 } \\
-/-\end{array}$} & 1 & 16 & 2 & none & none & paluse & -0.079181246 & -0.079181246 \\
\hline & 3 & 13 & 7 & none & Both cataract & palse & 0 & 0.15490196 \\
\hline & 6 & 17 & 6 & none & none & $\begin{array}{l}\text { STTA(40 } \\
\text { mg),paluse }\end{array}$ & 1 & 0.301029996 \\
\hline & 7 & 20 & 1 & none & none & paluse & -0.176091259 & 0 \\
\hline & 15 & 4 & 4 & none & none & PSL30mg & -0.176091259 & -0.176091259 \\
\hline & 24 & 42 & 4 & none & none & paluse & -0.176091259 & -0.176091259 \\
\hline$P$-value & & $0.586^{*}$ & $0.87^{*}$ & & & & $0.645^{*}$ & $0.301^{*}$ \\
\hline
\end{tabular}

STTA sub-tenon triamcinolone acetonide

treatment has always been used. But future treatments for recurrent VKH may be changed [15].

Previous studies have reported that HLAs represent a set of cell surface molecules mediating leukocyte interactions. HLA therefore plays an important role in immune system function as well as in the pathogenesis of autoimmune diseases, including VKH. Almost 40 years ago, an association between HLA-BW22J and VKH was reported [16]. Since then, more articles have been published regarding the associations of different HLA types to VKH. Among these, most investigations have focused on the HLA-DR4 serotype and its corresponding allele, HLA-DRB1*04 [17]. Shi et al. 

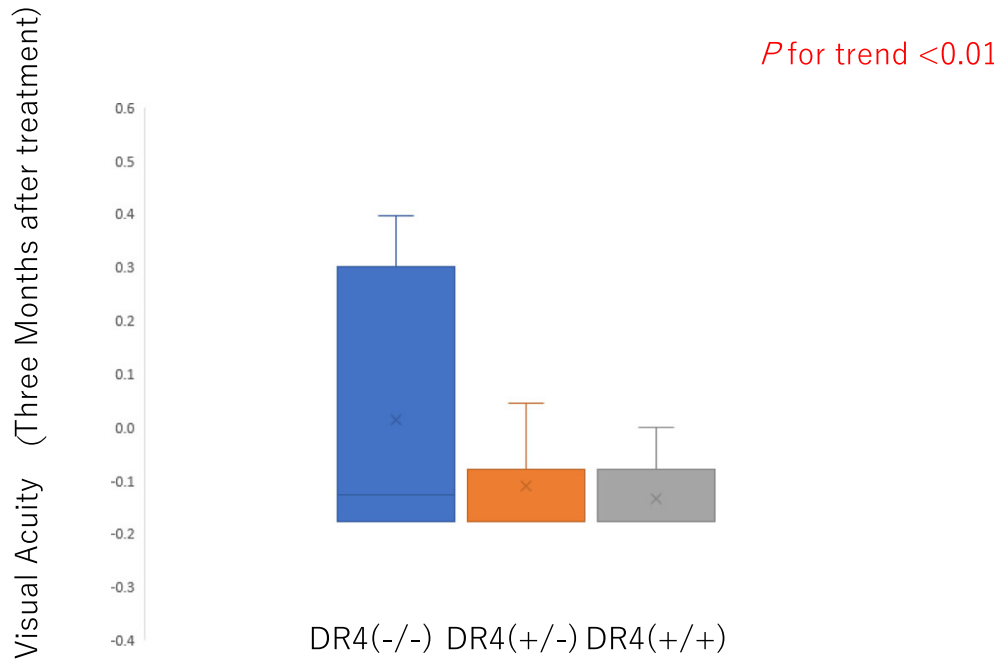

Fig. 2 Linear regression in best-corrected visual acuity (BCVA) at final visit. LogMAR BCVA for Vogt-Koyanagi-Harada (VKH) disease at 3 months (after treatment) on linear regression among the three groups of homozygotes (HLA-DRB1*04+/+), heterozygotes (HLA-DRB1*04+/-), and normal subjects (HLA-DRB1*04-/-)

reported a meta-analysis confirming the association between VKH and HLA-DR4/DRB1*04, finding that the strength of association differed between ethnic groups, and identifying HLA-DRB1*0404, 0405, and 0410 as risk suballeles, and 0401 as a protective sub-allele $[18,19]$.

For groups not having HLA-DR4/DRB1*04, the diagnosis may be not typical for VKH. However, both homozygous and heterozygous groups showed findings above the regression equation line, and were considered to have certain statistical meaning.

In the study of type 1 diabetes in Japanese, disease susceptibility has been found to differ between homozygotes and heterozygotes [20]. Similarly, disease susceptibility to VKH may differ between homozygotes and heterozygotes. Both before and after steroid treatment, homozygotes displayed the best post-treatment visual acuity. Normal subjects (no HLA-DRB1*04 allele) showed the poorest visual acuity after treatment. This indicates that therapeutic response and sensitivity to steroid treatment may depend on allele HLA-DRB1*04. An understanding of the pathogenic conditions that must exist to explain these results is difficult to reach. However, previous reports using methods such as haplotype linkage disequilibrium have suggested associations with genes closely related to immunity and inflammation, such as the IKBL gene and TNFA gene present in HLA class III [21-23].

The HLA-DRB1*04 allele is known to represent a diseaseassociated gene that is frequently found in $\mathrm{VKH}$, but our study suggested the possibility of disease resistance in association with this allele. On the other hand, another report found that presence of the HLA-DRB1\%04 allele was related to the prolongation of $\mathrm{VKH}$ in Japanese patients [24].
We know that the HLA-DRB1*04 allele is the key to $\mathrm{VKH}$, but the details remain elusive. According to the previous report, CCT correlates with vision prognosis in VKH [25]. However, in this case series with possible problems in the limited number of cases, the correlation between allele HLA-DRB1*04 and CCT could not be determined. Another limitation is following periods had variations and the bias cannot be denied completely.

\section{Conclusion}

We report that alleles of HLA-DRB1*04 might affect visual prognosis and be related to early response after initiation of treatment in $\mathrm{VKH}$ disease. In the future, the complete genetic predisposition of $\mathrm{VKH}$ is expected to be elucidated, leading to the development of nextgeneration treatments and preventive measures. We acknowledge these potential issues, as well as the need for future worldwide studies into the correlation between the HLA-DRB1*04 allele and visual outcomes in VKH.

\section{Authors' contributions}

MT wrote the main text of the manuscript and prepared the figures. Datasets were prepared by MT and NM, TK, and SH reviewed the manuscript and checked the statistical analysis advice. All authors have read and approved the manuscript in final state.

\section{Funding}

None of the authors have any proprietary or financial interests to declare.

Availability of data and materials

The data generated and/or analyzed for this study is available from the corresponding author upon request.

Ethics approval and consent to participate

All procedures performed in studies involving human participants were conducted in accordance with the ethical standards of the Institutional and/ 
or National Research Committee and with the 1964 Declaration of Helsinki and its later amendments or comparable ethical standards.

Approval for this study was obtained prior to the start of the study from the institutional review board at Osaka City University, Japan (IRB-4239). Written informed consent for the storage of patient information in the hospital database and use in the research was provided by all patients enrolled in the study.

\section{Consent for publication}

Not Applicable.

\section{Competing interests}

The authors declare that they have no competing interests.

Received: 4 July 2019 Accepted: 28 October 2019

Published online: 07 November 2019

\section{References}

1. Greco A, Fusconi M, Gallo A, Turchetta R, Marinelli C, Macri GF, et al. VogtKoyanagi-Harada syndrome. Autoimmun Rev. 2013;12:1033-8.

2. Ohno S, Minakawa R, Matsuda H. Clinical studies of Vogt-Koyanagi-Harada's disease. Jpn J Ophthalmol. 1988;32:334-43.

3. Rutzen AR, Ortega-Larrocea G, Schwab IR, Rao NA. Simultaneous onset of Vogt-Koyanagi-Harada syndrome in monozygotic twins. Am J Ophthalmol. 1995;119:239-40.

4. Caillat-Zucman S. Molecular mechanisms of HLA association with autoimmune diseases. Tissue Antigens. 2009;73:1-8.

5. Davis JL, Mittal KK, Freidlin V, Mellow SR, Optican DC, Palestine AG, et al. HLA associations and ancestry in Vogt-Koyanagi-Harada disease and sympathetic ophthalmia. Ophthalmology. 1990;97:1137-42.

6. Kim MH, Seong MC, Kwak NH, Yoo JS, Huh W, Kim TG, et al. Association of HLA with Vogt-Koyanagi-Harada syndrome in Koreans. Am J Ophthalmol. 2000;129:173-7.

7. Nakayama M, Keino H, Watanabe T, Okada AA. Clinical features and visual outcomes of 111 patients with new-onset acute Vogt-Koyanagi-Harada disease treated with pulse intravenous corticosteroids. Br J Ophthalmol. 2019;103:274-8.

8. Urzua CA, Velasquez V, Sabat P, et al. Earlier immunomodulatory treatment is associated with better visual outcomes in a subset of patients with VogtKoyanagi-Harada. Acta Ophthalmol. 2015;93:475-80.

9. Ng JY, Luk FO, Lai TY, Pang CP. Influence of molecular genetics in VogtKoyanagi-Harada disease. J Ophthalmic Inflamm Infect. 2014;4:20.

10. Urzua CA, Chen P, Chaigne-Delalande B, Liu B, Anguita R, Guerrero J, et al. Glucocorticoid receptor a and MKP-1 as candidate biomarkers for treatment response and disease activity in Vogt-Koyanagi-Harada disease. Am J Ophthalmol. 2019;50002-9394(19):30324-1.

11. Sugiura S. Vogt-Koyanagi-Harada disease. Jpn J Ophthalmol. 1978;22:9-35.

12. Read RW, Holland GN, Rao NA, Tabbara KF, Ohno S, Arellanes-Garcia L, et al. Revised diagnostic criteria for Vogt-Koyanagi-Harada disease: report of an international committee on nomenclature. Am J Ophthalmol. 2001;131:647-52.

13. Peduzzi $\mathrm{P}$, Concato J, Kemper E, Holford TR, Feinstein AR. A simulation study of the number of events per variable in logistic regression analysis. J Clin Epidemiol. 1996;49:1373-9.

14. Hirooka K, Saito W, Namba K, Mizuuchi K, Iwata D, Hashimoto Y, Ishida S. Significant role of the choroidal outer layer during recovery from choroidal thickening in Vogt-Koyanagi-Harada disease patients treated with systemic corticosteroids. BMC Ophthalmol. 2015;15:181.

15. Rosenbaum JT. Uveitis: contrasting the approaches in Japan and the United States. Jpn J Ophthalmol. 2019;63:1-6.

16. Olerup $\mathrm{O}$, Zetterquist $\mathrm{H}$. HLA-DR typing by PCR amplification with sequence-specific primers (PCR-SSP) in 2 hours: an alternative to serological DR typing in clinical practice including donor-recipient matching in cadaveric transplantation. Tissue Antigens. 1992;39:225-35.

17. Tagawa Y, Sugiura S, Yakura H, Wakisaka A, Aizawa M. Letter: HLA and VogtKoyanagh-Harada syndrome. N Engl J Med. 1976;295:173.

18. Shindo Y, Ohno S, Yamamoto T, Nakamura S, Inoko H. Complete association of the HLA-DRB1*04 and -DQB1*04 alleles with Vogt-Koyanagi-Harada's disease. Hum Immunol. 1994;39:169-76.
19. Shi T, Lv W, Zhang L, Chen J, Chen H. Association of HLA-DR4/HLA-DRB1*04 with Vogt-Koyanagi-Harada disease: a systematic review and meta-analysis. Sci Rep. 2014;4:6887.

20. Kawabata Y, Ikegami H, Kawaguchi Y, Fujisawa T, Shintani M, Ono M, Nishino M, Uchigata Y, Lee I, Ogihara T. Asian-specific HLA haplotypes reveal heterogeneity of the contribution of HLA-DR and -DQ haplotypes to susceptibility to type 1 diabetes. Diabetes. 2002;51:545-51.

21. Allcock RJ, Christiansen FT, Price P. The central MHC gene IKBL carries a structural polymorphism that is associated with HLA-A3,B7,DR15. Immunogenetics. 1999;49:660-5.

22. Allcock RJ, de la Concha EG, Fernandez-Arquero M, Vigil P, Conejero L, Arroyo R, Price P. Susceptibility to multiple sclerosis mediated by HLA-DRB1 is influenced by a second gene telomeric of the TNF cluster. Hum Immunol. 1999;60:1266-73.

23. Chinoy H, Li CK, Platt H, Fertig N, Varsani $\mathrm{H}$, Gunawardena H, Betteridge Z, Oddis CV, McHugh NJ, Wedderburn LR, Ollier WE, Cooper RG, Adult Onset Myositis Immunogenetic Consortium UK, UK Juvenile Dermatomyositis Research Group. Genetic association study of NF-KB genes in UK Caucasian adult and juvenile onset idiopathic inflammatory myopathy. Rheumatology (Oxford). 2012;51(5):794-9.

24. Islam SM, Numaga J, Matsuki K, Fujino Y, Maeda H, Masuda K. Influence of HLA-DRB1 gene variation on the clinical course of Vogt-Koyanagi-Harada disease. Invest Ophthalmol Vis Sci. 1994;35:752-6.

25. Nakayama M, Keino H, Okada AA, Watanabe T, Taki W, Inoue M, Hirakata A. Enhanced depth imaging optical coherence tomography of the choroid in Vogt-Koyanagi-Harada disease. Retina. 2012;32:2061-9.

\section{Publisher's Note}

Springer Nature remains neutral with regard to jurisdictional claims in published maps and institutional affiliations.

Ready to submit your research? Choose BMC and benefit from:

- fast, convenient online submission

- thorough peer review by experienced researchers in your field

- rapid publication on acceptance

- support for research data, including large and complex data types

- gold Open Access which fosters wider collaboration and increased citations

- maximum visibility for your research: over $100 \mathrm{M}$ website views per year

At BMC, research is always in progress.

Learn more biomedcentral.com/submissions 